\title{
Co-Occurring CSF3R W791* Germline and Somatic T618I Driver Mutations Induce Early CNL and Clonal Progression to Mixed Phenotype Acute Leukemia
}

\author{
Franziska C. Adam ${ }^{1,+}{ }^{\text {, Jakub Szybinski }}{ }^{2,+}{ }^{+}$Jörg P. Halter ${ }^{1} \mathbb{D}$, Nathan Cantoni ${ }^{3}$, Friedel Wenzel ${ }^{4}$, \\ Katharina Leonards ${ }^{1}$ (D), Sime Brkic ${ }^{2}$, Jakob R. Passweg ${ }^{1}{ }^{(D)}$, Ivo Touw ${ }^{5}$, Julia E. Maxson ${ }^{6}$ and Sara C. Meyer ${ }^{1,2, *}$
}

Citation: Adam, F.C.; Szybinski, J.; Halter, J.P.; Cantoni, N.; Wenzel, F.; Leonards, K.; Brkic, S.; Passweg, J.R.; Touw, I.; Maxson, J.E.; et al. Co-Occurring CSF3R W791* Germline and Somatic T618I Driver Mutations Induce Early CNL and Clonal Progression to Mixed Phenotype Acute Leukemia. Curr. Oncol. 2022, 29, 805-815. https:// doi.org/10.3390/curroncol29020068

Received: 31 December 2021

Accepted: 28 January 2022

Published: 1 February 2022

Publisher's Note: MDPI stays neutral with regard to jurisdictional claims in published maps and institutional affiliations.

Copyright: (C) 2022 by the authors. Licensee MDPI, Basel, Switzerland. This article is an open access article distributed under the terms and conditions of the Creative Commons Attribution (CC BY) license (https:// creativecommons.org/licenses/by/ $4.0 /)$.
1 Division of Hematology, University Hospital Basel, CH-4031 Basel, Switzerland; franziska.adam@usb.ch (F.C.A.); joerg.halter@usb.ch (J.P.H.); katharina.leonards@usb.ch (K.L.); jakob.passweg@usb.ch (J.R.P.)

2 Department of Biomedicine, University Hospital Basel and University of Basel, CH-4031 Basel, Switzerland; jakub.szybinski@unibas.ch (J.S.); sime.brkic@unibas.ch (S.B.)

3 Kantonsspital Aarau, CH-5001 Aarau, Switzerland; nathan.cantoni@ksa.ch

4 Institute of Medical Genetics and Pathology, University Hospital Basel, CH-4031 Basel, Switzerland; friedel.wenzel@usb.ch

5 Department of Hematology, Erasmus University Medical Center, 3015 CN Rotterdam, The Netherlands; i.touw@erasmusmc.nl

6 Knight Cancer Institute, Oregon Health \& Science University, Portland, OR 97239, USA; maxsonj@ohsu.edu

* Correspondence: sara.meyer@unibas.ch; Tel.: +41-(61)-5565965

+ These authors contributed equally to this work.

\begin{abstract}
Chronic neutrophilic leukemia (CNL) relates to mutational CSF3R activation with membrane proximal CSF3R mutations such as T618I as driver mutations, but the significance of truncating mutations is not clarified. In CNL, concomitant mutations promote disease progression, but insight into longitudinal acquisition is incomplete. In this study, we investigated the role of co-occurring germline and somatic CSF3R mutations in CNL, and assessed the impact of clonal evolution on transformation to acute leukemia. We employed sequential next generation sequencing and SNP array karyotyping to assess clonal evolution in CNL of early manifestation age based on a 33-year-old patient. Germline vs. somatic mutations were differentiated using a sample from the hair follicle. To investigate a potential predisposition for CNL development and progression by germline CSF3RW791*, allelic localizations were evaluated. We detected a somatic CSF3R-T618I mutation at $46 \%$ variant allele frequency (VAF) at the time of CNL diagnosis, which co-occurred with a CSF3R-W791* truncation at 50\% VAF in the germline. Evaluation of allelic localization revealed CSF3R-T618I and W791* on the same allele. A concomitant ASXL1 mutation at 39\% VAF increased to $48 \%$ VAF upon transformation to mixed phenotype acute leukemia (MPAL), which has both myeloid and lymphoid features. Clonal evolution further involved expansion of the CSF3R double-mutant clone to $90 \%$ VAF via copy neutral loss of heterozygosity on chromosome $1 \mathrm{p}$ and the emergence of a RUNX1 mutant subclone. Allogeneic transplantation induced complete remission. This study highlights that CNL not only transforms to AML but also to MPAL. The molecular evolution is especially interesting with a CSF3R-W791* mutation in the germline and acquisition of CSF3R-T618I on the same allele compatible with increased susceptibility for mutation acquisition facilitating RUNX1-related clonal transformation.
\end{abstract}

Keywords: CSF3R; clonal evolution; chronic neutrophilic leukemia; mixed phenotype acute leukemia

\section{Introduction}

Chronic neutrophilic leukemia (CNL) is a myeloproliferative neoplasm characterized by the overproduction of neutrophils and activating mutations in CSF3R, the receptor for 
colony stimulating factor 3 (GCSF). Presentation is heterogeneous ranging from asymptomatic clonal neutrophilia to severe constitutional symptoms, splenomegaly, bleeding diathesis and an inherent risk for transformation to acute myeloid leukemia (AML) [1]. Activating point mutations in CSF3R, most prevalently CSF3R T618I, were discovered in 2013 and recognized as driver mutation in a majority of CNL patients [2,3]. These activating mutations localize to a membrane proximal, extracellular portion of the CSF3R and induce ligand independent receptor activation resulting in constitutive activation of the JAK-STAT signaling pathway. Their essential role for CNL pathogenesis has led to the implementation as key genetic biomarker into the WHO diagnostic criteria for $\mathrm{CNL}$, which greatly facilitated CNL diagnosis along with neutrophilia and hypercellular marrow with increased granulopoiesis [4,5]. In line with JAK-STAT pathway activation, efficacy of the JAK1/2 inhibitor ruxolitinib has been demonstrated in case reports and a clinical trial for patients with CNL and atypical CML, a related myeloproliferative disorder [2,6]. Rarely, CSF3R activating mutations such as T618I, T640N and N610H have also been detected in germline in individuals with congenital neutrophilia [7-9].

Genetic characterization of CSF3R in CNL has also revealed nonsense or frameshift mutations in more distal, intracellular portions of CSF3R resulting in receptor truncation [2]. It has been shown that this second class of CSF3R mutations increases cell surface expression of CSF3R, mediating ligand hypersensitivity [10]. Notably, a minority of CSF3RT618I mutant CNL cases also show a concomitant CSF3R truncation mutation. It is not entirely clear how activating and truncating CSF3R co-mutations alter progression to acute leukemia, although truncating mutations can enhance CSF3R-T618I-mediated growth in some in vitro assays [11]. Germline CSF3R cytoplasmic truncation mutations have not been reported thus far.

Transformation to acute leukemia is reported in $10-21 \%$ of CNL patients and median time to transformation is approximately 21 months [1]. Mutations in the epigenetic regulator ASXL1, which are frequently detected along with CSF3R mutations at CNL diagnosis, confer adverse prognosis and increased risk of transformation [12]. However, the molecular dynamics of clonal evolution and progression of CNL to AML is incompletely understood [13]. Comprehensive genomic and transcriptomic profiling of CNL patients revealed multiple co-occurring mutations, which are enriched in CNL, including ASXL1, SRSF2, SETBP1, TET2, EZH2, U2AF1 and others [13]. To date, specific insight into clonal evolution and transformation of CNL patients is incomplete and particularly challenging given the rarity of this disease. Detailed molecular characterization is warranted to enable improved prognostication and inform novel therapeutic approaches.

CSF3R truncation mutations are also prevalent in patients with severe congenital neutropenia ( $\mathrm{SCN}$ ), a condition with heterogenous basis often related to mutations in the ELANE gene encoding neutrophil elastase [14]. While SCN patients are often managed by long-term administration of G-CSF, the presence of CSF3R truncation mutations associated with increased risk of progression from SCN to AML $[15,16]$. Studies of SCN clonal evolution have typically shown the emergence of subclones with RUNX1, ASXL1 or SUZ12 mutations [17]. More rarely, acquisition of activating point mutations in CSF3R as e.g., T618I has also been seen [18]. Thus, CSF3R truncating and activating mutations may co-occur in SCN similarly to CNL, but while activating CSF3R mutations are present in the vast majority of $\mathrm{CNL}$ patients with a minority co-carrying truncation mutants, truncating CSF3R mutations prevail in SCN [18]. This may in part relate to the differentiation potential of the two classes of mutations, while CSF3R-T618I seems to maintain full differentiation potential, CSF3R truncating mutations impair differentiation [19]. Here we investigate the role of co-occurring CSF3R germline and somatic mutations and clonal evolution in CNL for transformation to acute leukemia based on a patient with early onset CNL. We report for the first time CNL transformation to acute leukemia of mixed phenotype (MPAL type) in the setting of a germline CSF3R-W791* truncation mutation located on the same allele as CSF3R-T618I, suggesting increased susceptibility for RUNX1-related clonal transformation. 


\section{Materials and Methods}

\subsection{Mutational Analysis by Next Generation Sequencing}

Mutational status was assessed using a 39-gene targeted AmpliSeq NGS assay covering full genes including splice sites covering $A B L 1, A S X L 1, B C R, B R A F, C A L R, C B L, C E B P A$, CHEK2, CSF3R, DNMT3A, EGLN1, EPOR, ETNK1, ETV6, EZH2, FLT3, GATA2, IDH1, IDH2, JAK2, KIT, KRAS, MPL, NF1, NPM1, NRAS, PDGFRA, PDGFRB, PTPN11, RUNX1, SETBP1, SF3B1, SH2B3, SRSF2, TET2, TP53, U2AF1, VHL, ZRSR2. A total of $60 \mathrm{ng}$ DNA extracted from whole bone marrow cells was amplified with 15 PCR cycles during automated library preparation with the Ion AmpliSeq Kit for Chef DL8 (Cat. A29024) on the IonChef instrument. Library pools were quantified by qPCR using the Ion Universal Quantitation Kit (Cat. A26217) and 30 pmol of the pool was sequenced on 530 Chips on an Ion S5XL sequencer. Sequences were aligned to the human reference genome GRCh37/hg19 and analyzed with IonReporter version 5.12 with a sensitivity limit of variant allelic frequency of $2-5 \%$. For analysis of germline mutations, $60 \mathrm{ng}$ DNA extracted from hair follicles was processed analogously as described above. Variant allele frequencies were plotted with GraphPadPrism9. Figure 1 was created with Biorender software (Biorender.com, last accession date 1 November 2021).

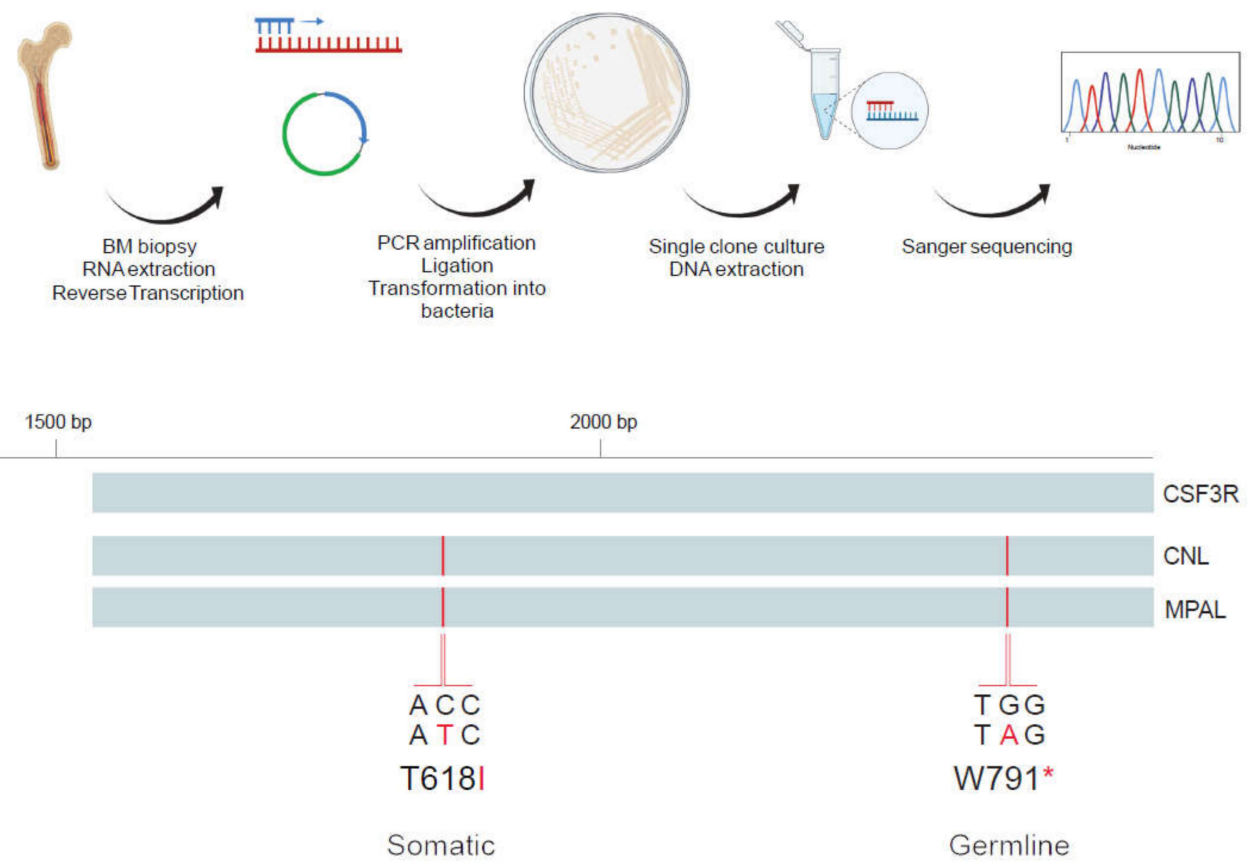

Figure 1. Allelic localization of germline CSF3R W791* and acquired CSF3R T618I in CNL and clonal progression to acute leukemia of mixed phenotype (MPAL type). Patient bone marrow cDNA from the CSF3R gene locus encompassing both T618I and W791* was amplified by PCR and Sanger sequencing of plasmid-emergent colonies to evaluate allelic localization (upper panel). CSF3R reference sequence (bottom panel, top line) was aligned with patient-derived DNA sequences at the stage of CNL (bottom panel, middle line) and of mixed phenotype acute leukemia (MPAL, bottom panel, bottom line). Sequence analyses demonstrated co-localization of T618I and W791* mutations (red vertical lines) in cis on the same allele at CNL and MPAL stages.

\subsection{Analysis of Allelic Localization of CSF3R Mutations by Sequencing of Plasmid DNA Clones}

The CSF3R gene region spanning both activating T618I and truncating W791* mutations was amplified by PCR from $4 \mu \mathrm{g}$ of bone marrow derived cDNA using CSF3R forward (5'-ACCATGGGACCCTCCCAGCA-3') and reverse primers (5'-AAGCTCCCCAGCGCCTCCAT $-3^{\prime}$ ) and Platinum ${ }^{\mathrm{TM}}$ Taq DNA Polymerase (Invitrogen, Waltham, MA, USA). Resulting PCR products were separated by agarose gel electrophoresis and gel extraction of PCR products of desired band-size was performed using Qiaquick Gel Extraction kit (Qiagen, 
Venlo, The Netherlands). Amplified CSF3R fragments were cloned into pCR4-TOPO vector and transformed into One Shot ${ }^{\mathrm{TM}}$ TOP10 E. coli bacteria (Invitrogen), which were plated on agar plates supplemented with ampicillin and cultured overnight at $37^{\circ} \mathrm{C}$. Single bacteria colonies were picked and expanded in LB-medium cultures. DNA was extracted by Minipreparation of plasmid DNA (Qiagen), processed by restriction enzyme digestion with EcoRI HF ${ }^{\circledR}$ (New England BioLabs, Ipswich, MA, USA), run on agarose gel and analyzed for presence of the CSF3R insert. TOPO M13 reverse primer was added to DNA from clones with CSF3R insert and 6 clones per disease stage were subjected to Sanger sequencing. Resulting sequences were aligned to CSF3R transcript variant 1 (NCBI Reference Sequence: NM_000760.4) and analyzed using Benchling Biology Software (2021), retrieved from https:/ / benchling.com (last accession date 16 June 2021).

\subsection{SNP Array Karyotyping}

DNA was extracted from bone marrow by MagnaPur (Roche, Basel, Switzerland) or Prepito-D system (PerkinElmer, Waltham, MA, USA) and DNA blood 600 kit (Chemagen, Baesweiler, Germany) and hybridized to Cytoscan HD array (Affymetrix, Santa Clara, CA, USA) according to manufacturer's instructions. Data were analyzed using the Chromosome Analysis Suite (ChAS) software (Affymetrix). Copy number variants (CNV) were considered with a size above $100 \mathrm{~kb}$; copy neutral loss of heterozygosity $(\mathrm{CN}-\mathrm{LOH})>5 \mathrm{Mb}$ and extending to telomeres is defined as acquired abnormality. All CNV and CN-LOH fulfilling these criteria were considered for this analysis and validated by visual inspection as well as annotated for size, position and location of genes based on the human genome version 19 (hg19) of USCS Genome Browser.

\section{Results}

\subsection{Early Onset CNL Demonstrates Progression to Mixed Phenotype Acute Leukemia}

CNL was diagnosed in a 33-year-old male in the absence of comorbidities or neoplastic disorders in his relatives according to WHO criteria (Table 1). Initial presentation was with symptomatic splenomegaly, shortness of breath, elevated white blood cell count (WBC) of $53 \times 10^{9} / \mathrm{L}$ with pronounced neutrophilia of $88 \%$ and mild anemia. Bone marrow was hypercellular with greatly increased and maturing granulopoiesis. $B C R-A B L 1$ fusion was negative while CSF3R mutations in CNL had yet to be discovered at the time of CNL diagnosis.

Cytoreductive treatment with hydroxyurea led to hematologic remission. Upon progressive neutrophilia and splenomegaly 5 years later, JAK $1 / 2$ inhibitor therapy with ruxolitinib was initiated resulting in a favorable clinical response and was intermittently switched to pegylated interferon alpha given the patient's wish to father children.

After a stable ten-year course, sudden clinical deterioration occurred, and diagnosis of acute leukemia was made. Leukemic blasts with extensive marrow infiltration were partially positive for cytochemical peroxidase staining (20-30\%), while Auer rods were absent, and immunophenotypic characterization confirmed co-existence of a myeloid CD34+, CD117+/-, HLA-DR+, cyMPO+/-, CD13+, CD33+, CD36+, CD71+ blast population negative for CD3-, cyCD3, CD19 and CD79a alongside a CD19+, CD22+/-, cyCD79a+, nuTDT+ und cyCD3- B-lymphoid blast population consistent with mixed phenotype acute leukemia (MPAL type B/myeloid) according to WHO criteria. To our knowledge, this represents the first report of CNL transforming to acute leukemia of MPAL type. After induction chemotherapy and consolidation by allogeneic hematopoietic stem cell transplantation (HSCT), the patient achieved continuous complete remission until the last follow-up, 24 months post-transplant. The patient's mother and father have deceased (due to nonhematologic disorders), but no specific information is available on hematologic aberrancies in the patient's parents or six siblings. 
Table 1. Pre-diagnostic, diagnostic and transformation phase of a patient with early onset CNL upon co-mutant CSF3R germline truncation and acquired activating mutation.

\begin{tabular}{|c|c|c|}
\hline Variation & Phase & Treatment \\
\hline Pre-diagnosis & $\begin{array}{l}\text { Germline CSF3R } \\
\text { truncation mutation } \\
\text { W791* }\end{array}$ & - $\quad$ none \\
\hline Diagnosis & $\mathrm{CNL}$ & $\begin{array}{l}\text { - } \quad \text { 1st line: Hydroxyurea } \\
\text { - } \\
\text { snd line: Ruxolitinib (progressive } \\
\text { - } \quad \text { 3rd line: Pegylated interferon alpha } \\
\text { (wish for children) }\end{array}$ \\
\hline Transformation & $\begin{array}{l}\text { Acute leukemia of } \\
\text { MPAL type }\end{array}$ & $\begin{array}{l}\text { Induction chemotherapy with } \\
\text { Cytarabine + Idarubicin (1 cycle) } \\
\text { followed by azacytidine } \\
\text { Allogeneic HSCT from HLA-identical } \\
\text { sibling after reduced intensity } \\
\text { conditioning }\end{array}$ \\
\hline Last follow-up & $\begin{array}{l}\text { Complete molecular } \\
\text { remission }\end{array}$ & - $\quad$ None \\
\hline
\end{tabular}

\subsection{Characterization of CSF3R Mutations Reveals W791* in Germline and in Cis with Somatic T618I}

Given the particular features of transformation to acute leukemia in this early onset CNL, we hypothesized that specific genetic factors could cooperate to promote emergence of CNL at early age and ultimately promote progression to acute leukemia. Thus, we investigated CSF3R gene mutations as well as the dynamics of clonal evolution by a sequential NGS approach using a 39-myeloid gene panel routinely used in our department. We detected the CSF3R T618I mutation at a variant allele frequency (VAF) of $46 \%$ and ASXL1 C759fs mutation at 39\% at CNL diagnosis in line with a high prevalence of ASXL1 mutations in CNL. Of note, we identified a co-occurrent CSF3R W791* truncation mutation at $50 \%$ VAF suggestive of germline origin. Analysis of germline DNA from hair follicle confirmed CSF3R W791* truncation mutation as a likely germline variant at 50\% VAF (Supplementary Figure S1). This finding adds new insight into CSF3R biology and contrasts with previous reports of rare germline CSF3R mutations identified in individuals with congenital neutrophilia, which so far exclusively represent CSF3R missense but not truncation mutations.

We next sought to establish whether a CSF3R germline truncation mutant could be involved in setting the stage and contribute to predisposition for CNL and leukemia development. To address this aspect, we evaluated the allelic localization of the co-occurring CSF3R auto-activating and truncation mutations. Patient-derived bone marrow DNA was amplified using a primer set that encompasses both mutations of interest, and was processed using a cloning approach and subjected to classical Sanger sequencing to assess for co-localization of the CSF3R T618I and W791* stop mutations on the same allele. Processed clones from CNL diagnosis revealed T618I and W791* mutations in cis on the identical allele (Figure 1). This was confirmed in leukemic blasts at the time-point of transformation with both mutations consistently located in cis in 6/6 analyzed clones. This finding is compatible with the notion of the CSF3R W791* germline variant mediating a potential predisposition for the acquisition of the CSF3R T618I driver mutation, thus facilitating early onset of CNL. 


\subsection{Clonal Progression Driven by CSF3R LOH and RUNX1 Mutation}

To further evaluate the factors precipitating transformation in the CSF3R double mutant setting, we performed in-depth genetic analyses including NGS and SNP array karyotyping at several time-points during disease evolution. We observed copy neutral loss of heterozygosity $(\mathrm{CN}-\mathrm{LOH})$ at chromosome $1 \mathrm{p}$ at the time of transformation to acute leukemia (Figure 2), which substantiated the CSF3R co-mutated clone to variant allele frequencies $>90 \%$ for both CSF3R mutations (Figure 3).

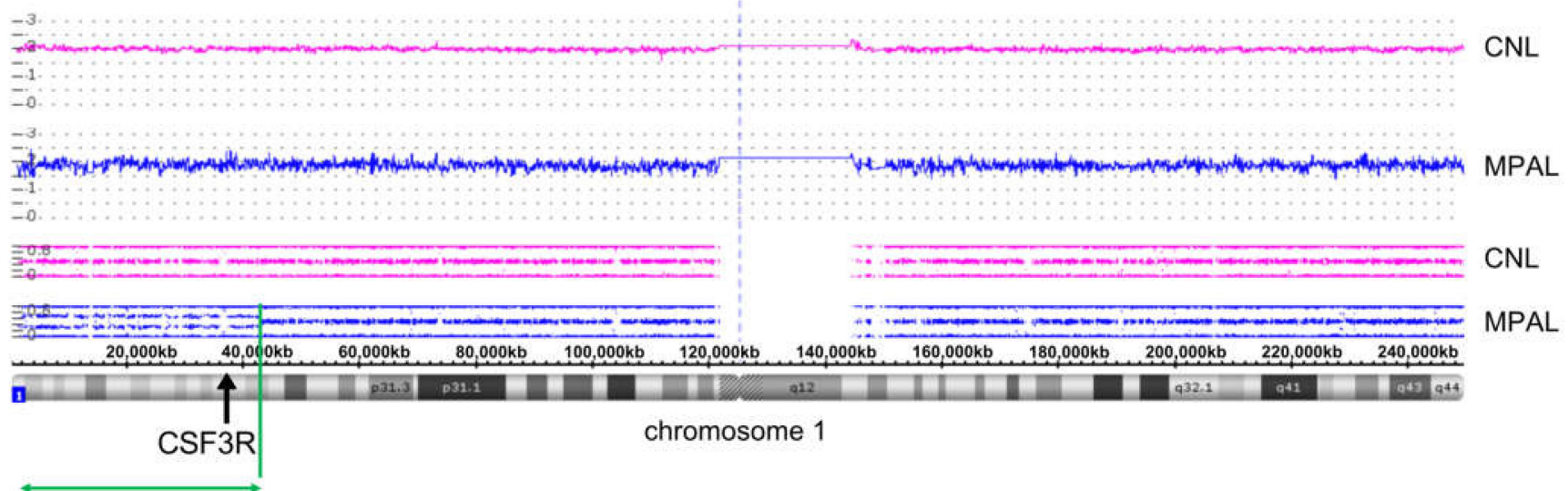

$\mathrm{CN}-\mathrm{LOH}$

Figure 2. Copy neutral loss of heterozygosity $(\mathrm{CN}-\mathrm{LOH})$ reflected by SNP array karyotyping upon CNL progression to acute leukemia of mixed phenotype (MPAL type). Purple color represents DNA from CNL phase, blue color represents DNA from time-point of transformation to acute leukemia of MPAL type. Upper panels show a smooth signal distribution for DNA from CNL and MPAL stage indicating continuous and unchanged copy number of 2 for both samples. Middle panels show B-allele frequency (BAF) distributions with DNA from CNL phase (purple) with normal "threeline pattern" indicating no $\mathrm{LOH}$, and DNA from MPAL phase (blue) with "four-line pattern" from cytogenetic bands 1p36.33 to 1p34.2 (molecular position: 849' 466 and 42'465'700) indicating loss of heterozygosity (LOH) in the affected region in mosaic status (highlighted by green arrow) at MPAL stage highlighting a persisting significance of CSF3R activation at transformation. The chromosome 1 ideogram is shown in the bottom panel. The combination of deviating LOH pattern along with copy number of 2 defines copy neutral loss of heterozygosity (CN-LOH). Black arrow indicates the CSF3R locus at 1 p34.3 (36 $\left.931^{\prime} 644-36^{\prime} 948^{\prime} 879\right)$, the vertical dotted blue line indicates the position of the centromere.

Also, the ASXL1 mutant subclone expanded, and in addition a RUNX1 R201Q mutation emerged with a high VAF of $75 \%$ relating to a mosaic trisomy 21 (Figure 3, Supplementary Figure S2). These findings suggest a persisting impact of CSF3R activation on transformation to acute leukemia, which is further complemented by additional high-risk mutations such as in the RUNX1 gene. 


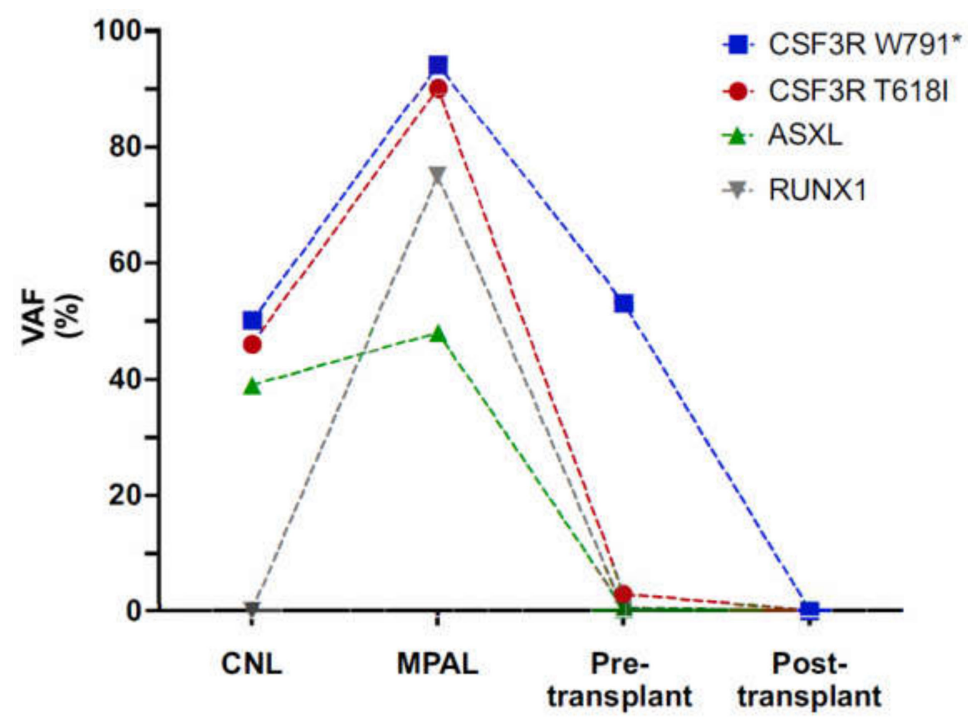

Figure 3. Clonal progression of CSF3R co-mutant CNL to acute leukemia of mixed phenotype (MPAL type) as reflected by sequential next generation sequencing. Upon CNL diagnosis, the germline CSF3R-W791* truncation mutation was complemented by a somatic CSF3R-T618I activating mutation at $46 \%$ variant allele frequency (VAF) along with an ASXL1 C759fs mutation at 39\% VAF. At transformation to mixed phenotype acute leukemia (MPAL), the CSF3R co-mutated clone had acquired an additional RUNX1 R201Q mutation at 75\% VAF. Mutant allele burden reflecting the MPAL clone was substantially reduced upon intensive induction therapy to CSF3R T618I 3\% VAF, ASXL1 C759fs 0.5\% VAF and RUNX1 R201Q 0.3\% VAF before allogeneic transplantation and was undetectable after allogeneic hematopoietic stem cell transplantation.

\section{Discussion}

The discovery of CSF3R mutations in the majority of CNL patients has identified constitutive activation of hematopoietic cytokine receptor signaling as essential to induce neutrophilia in CNL. This is analogous to other myeloproliferative neoplasms (MPN) including polycythemia vera (PV), essential thrombocythemia (ET) and primary myelofibrosis (PMF) [2]. Experimental studies in murine models have validated CSF3R activating mutations such as T618I as driver mutations that promote development. CSF3R-T618I has become an established biomarker facilitating CNL diagnosis and also provides a therapeutic target for treatment with the JAK1/2 inhibitor ruxolitinib [4-6].

$\mathrm{CNL}$ is a rare hematologic disorder with variable clinical course, but dismal prognosis upon transformation [20]. Thus, a deeper understanding of the molecular mechanisms underlying pathogenesis and transformation is required. So far, transformation of CNL to acute leukemia has been reported to present as acute myeloid leukemia (AML) [21], and recently, a first case of CNL transforming to B-lymphoblastic leukemia has been described [22]. Here we investigated early onset CNL based on a patient presenting with $\mathrm{CNL}$ at a young age of 33 years, who showed progression to acute leukemia of mixed phenotype (MPAL), which to our knowledge represents the first report of such manifestation. As CNL is rare, characterization of additional individuals with transformation to acute leukemia of MPAL-type will be required to see whether mixed phenotype acute leukemia particularly associates with early onset $\mathrm{CNL}$, and to confirm the importance of particular molecular alterations.

CSF3R truncation mutations in CNL primarily co-occur with auto-activating point mutations (such as T618I) and are rarely found in isolation [23]. While CSF3R truncation mutations promote leukemic transformation in severe congenital neutropenia (SCN), their significance for CNL pathogenesis has not been fully clarified. So far, CSF3R truncation mutations have not been reported in the germline. It is an intriguing question whether they would promote acquisition of additional, transformative CSF3R activating mutations or the expansion of clones with these mutations thus conferring predisposition states for $\mathrm{CNL}$ 
pathogenesis and progression. One case report on a child with a T618I germline mutation developing CNL with a somatic W791* mutation has been published [9]. To our knowledge, CNL based on germline CSF3R W791* along with an acquired T618I mutation has not been described thus far. Analyses of extensive family pedigrees would be required to assess leukemia predisposition based on CSF3R truncations, a resource which is unavailable.

The presence of both a CSF3R point and truncating mutation raises the question of whether they are on the same allele, and thus present in the same protein product. Previous sequencing of a very limited number of cases revealed that point and truncating mutations can occur on the same allele, but analysis of more cases is needed to know if they are usually present in cis [2]. Thus, we performed molecular analyses of the allelic localizations of the CSF3R truncation and activating mutations sequentially at the several stages of the disease. We investigated patient bone marrow derived DNA at CNL and MPAL stages and revealed localization of truncation and activating mutations on the same allele of the CSF3R gene. While this finding suggests a potential predisposition for CNL development and progression by the CSF3R W791* germline truncation mutation, more analogous cases will be required to corroborate such a pattern.

At both the initial diagnosis of CNL and subsequent progression to MPAL, a truncating mutation in the epigenetic regulator ASXL1 was observed. ASXL1 mutations are associated with the premalignant condition, clonal hematopoiesis, and are often thought to be early events in myeloid leukemogenesis [24]. Indeed, serial sequencing of samples from patients with CNL on ruxolitinib therapy revealed that the CSF3R mutant clone can shrink dramatically while the ASXL1 mutant clone remains, indicating that ASXL1 mutations were antecedent in those cases [25]. Interestingly, in this case, the ASXL1 mutant clone has a lower VAF than both CSF3R mutations at all timepoints studied, consistent with it being a later genetic event. This case highlights the multiple potential paths to CNL biogenesis.

We therefore sought to understand the molecular changes that enable the clonal evolution of CNL progressing to acute leukemia of MPAL type. NGS profiling along with SNP array karyotyping at several stages of disease progression revealed that clonal evolution was not halted but continued despite intermittent targeted inhibition of JAK2 signaling via the JAK1/2 inhibitor ruxolitinib, as has been reported for the classical, Philadelphia chromosome-negative MPN [26]. CN-LOH at chromosome 1p encompassing the CSF3R gene locus resulted in doubled CSF3R mutant allele burden for the activating T618I as well as the W791* truncation mutation, thus further enhancing receptor activation and overexpression upon progression. At the pre-transplant timepoint in which there is a near elimination of the CSF3R-T618I, ASXL1-C759fs and RUNX1-R201Q mutant clone, the variant allele frequency of CSF3R-W791* returns to near 50\% frequency, indicating that chemotherapy-induced clearance of the leukemic clone effectively restores the locus to heterozygosity (Figure 3). The persistence of the CSF3R W791* at 50\% VAF pre-transplant, further supports the idea that this clone was germline. This finding highlights the persistent role of CSF3R activation in CNL, and at the stage of transformation to acute leukemia of MPAL type. It further underscores the need for therapeutic approaches to target mutational CSF3R activation.

In addition to CN-LOH at the CSF3R locus, transformation to MPAL was also associated with the acquisition of a point mutation in $R U N X 1$, a transcription factor that regulates myeloid development. RUNX1 mutations are associated with poor prognosis in AML $[27,28]$. RUNX1 and CSF3R are frequently co-mutated in the progression to acute myeloid leukemia from SCN [18]. Neither acquisition of a RUNX1 point mutation or $\mathrm{CN}-\mathrm{LOH}$ at the CSF3R locus readily explain the mixed myeloid and lymphoid phenotype associated with transformation to acute leukemia.

MPAL is known to harbor various AML- and ALL-associated mutations. Characterization of the genetic basis of MPAL is still incomplete and a specific mutational pattern has not been consistently identified so far. Takahashi and colleagues [29], who described the genomic profiles of 13 adult patients with B/M MPAL, reported prevalent ASXL1 mutations (23\% of individuals) as well as RUNX1 mutations ( $46 \%$ of individuals) similar to the 
mutational pattern in our MPAL patient. Other mutations known to be prevalent in hematological malignancies including FLT3, NRAS or SRSF2 mutations have also been repeatedly reported in MPAL [30]. Of note, a landmark study by Alexander and colleagues, which analyzed mutational profiles of 35 patients, specifically identified ZNF384 rearrangements at high incidence (43\%) in children [31]. CSF3R mutations were found in two prior cases of early T-cell precursor acute lymphoblastic leukemia, in which the leukemic cells can also harbor both lymphoid and myeloid markers. However, in these cases the CSF3R mutations were accompanied by NOTCH1 mutations which are potent lymphoid drivers [2,32]. It is possible that another unidentified mutation promotes the mixed leukemic phenotype in this case, or that alternately the germline nature of this mutation causes leukemia associated mutations to be present in a more primitive and multipotent stem cell than what is typically associated with CNL. The exact cell of origin for CNL is still unknown.

\section{Conclusions}

The molecular characterization of this early onset CNL provides new insights into CNL biogenesis and highlights essential open issues which require evaluation to further enhance our understanding of CNL development and progression to enable development of more effective therapeutic strategies. CSF3R truncation mutations were shown to be sensitive to dasatinib via Src kinase inhibition, but it is not known whether co-targeting CSF3R truncations such as W791* by dasatinib would provide therapeutic benefit in CSF3R co-mutant cases along with ruxolitinib treatment. Furthermore, the intriguing question whether dasatinib would be beneficial in a germline truncation situation to avoid progression to CNL and acute leukemia remains unanswered. Further molecular studies of additional patients with CSF3R driven malignancies will be essential to elucidate these theories, and to shed light on the complex evolution of CNL.

Supplementary Materials: The following supporting information can be downloaded at: https: / / www.mdpi.com/article/10.3390/curroncol29020068/s1, Figure S1: Identification of germline origin of CSF3R-W791* but not T618I mutation, Figure S2: SNP array indicating mosaic trisomy 21 in the MPAL stage.

Author Contributions: F.C.A., J.S., J.P.H., F.W., J.E.M. and S.C.M. designed the study and wrote the paper. F.C.A., J.S., J.P.H., N.C., F.W., K.L., S.B., J.R.P., I.T., J.E.M. and S.C.M. performed experiments, analyzed data, or provided essential data or experimental materials. All authors have read and agreed to the published version of the manuscript.

Funding: This work was generously supported by research grants from Swiss National Science Foundation (PCEFP3_181357), the Swiss BRIDGE Foundation (PSB-4066-06-2016), the Cancer League Basel and the "Stiftung für krebskranke Kinder Regio Basiliensis" (KLbB-4784-02-2019) and the Foundation for the Fight against Cancer to SCM. JEM is supported by NIH/NHLBI R01 HL157147-01 and a Concern Foundation Conquer Cancer Now Award.

Institutional Review Board Statement: The study was conducted in accordance with the Declaration of Helsinki and assessed by the "Ethikkommission Nordwest- und Zentralschweiz (EKNZ)" responsible for the University Hospital Basel and the University of Basel, Switzerland (Req-2022-00077, 24 January 2022).

Informed Consent Statement: Informed consent was obtained from all subjects involved in the study.

Data Availability Statement: The data presented in this study are available on request from the corresponding author (sara.meyer@unibas.ch). The data are not publicly available due to privacy/ethical reasons.

Acknowledgments: Figure 1 was created with Biorender software (Biorender.com, last accession date 1 November 2021).

Conflicts of Interest: FCA holds shares in Novartis. NC has consulted for and received honoraria from AbbVie, Astra Zeneca, BMS/Celgene, Gilead, Novartis, OrphaSwiss, Pfizer, Sandoz, Sanofi-Aventis, Takeda/Shire, has received research support from Novartis, support for conference attendance from AbbVie, Amgen, BMS/Celgene, Gilead and Roche, and is on supervisory boards 
of AbbVie, Alexion, Amgen, BMS/Celgene, Gilead, Incyte, Janssen-Cillag, Novartis, OrphaSwiss, Pfizer, Roche, Sandoz, Sanofi-Aventis and Takeda/Shire. JEM receives research funding from Gilead Sciences and serves on a scientific advisory board for Ionis Pharmaceuticals. SCM has consulted for and received honoraria from Celgene/BMS and Novartis. All other authors do not have relevant conflicts of interest to declare.

\section{References}

1. Szuber, N.; Elliott, M.; Tefferi, A. Chronic neutrophilic leukemia: 2020 update on diagnosis, molecular genetics, prognosis, and management. Am. J. Hematol. 2020, 95, 212-224. [CrossRef] [PubMed]

2. Maxson, J.E.; Gotlib, J.; Pollyea, D.A.; Fleischman, A.G.; Agarwal, A.; Eide, C.A.; Bottomly, D.; Wilmot, B.; McWeeney, S.K.; Tognon, C.E. Oncogenic CSF3R Mutations in Chronic Neutrophilic Leukemia and Atypical CML. N. Engl. J. Med. 2013, 368, 1781-1790. [CrossRef] [PubMed]

3. Beekman, R.; Valkhof, M.; Van Strien, P.; Valk, P.J.; Touw, I.P. Prevalence of a new auto-activating colony stimulating factor 3 receptor mutation (CSF3R-T595I) in acute myeloid leukemia and severe congenital neutropenia. Haematologica 2013, 98, e62-e63. [CrossRef] [PubMed]

4. Arber, D.A.; Orazi, A.; Hasserjian, R.; Thiele, J.; Borowitz, M.J.; Le Beau, M.M.; Bloomfield, C.D.; Cazzola, M.; Vardiman, J.W. The 2016 revision to the World Health Organization classification of myeloid neoplasms and acute leukemia. Blood. Am. Soc. Hematol. 2016, 127, 2391-2405. [CrossRef] [PubMed]

5. Fleischman, A.; Maxson, J.E.; Luty, S.B.; Agarwal, A.; Royer, L.R.; Abel, M.L.; MacManiman, J.D.; Loriaux, M.M.; Druker, B.; Tyner, J.W. The CSF3R T618I mutation causes a lethal neutrophilic neoplasia in mice that is responsive to therapeutic JAK inhibition. Blood 2013, 122, 3628-3631. [CrossRef] [PubMed]

6. Dao, K.-H.T.; Gotlib, J.; Deininger, M.M.N.; Oh, S.T.; Cortes, J.E.; Collins, R.H.; Winton, E.F.; Parker, D.R.; Lee, H.; Reister, A.; et al. Efficacy of Ruxolitinib in Patients With Chronic Neutrophilic Leukemia and Atypical Chronic Myeloid Leukemia. J. Clin. Oncol. 2020, 38, 1006-1018. [CrossRef]

7. Plo, I.; Zhang, Y.; Le Couédic, J.-P.; Nakatake, M.; Boulet, J.-M.; Itaya, M.; Smith, S.O.; Debili, N.; Constantinescu, S.; Vainchenker, W.; et al. An activating mutation in the CSF3R gene induces a hereditary chronic neutrophilia. J. Exp. Med. 2009, 206, $1701-1707$. [CrossRef]

8. $\quad$ Spiciarich, D.R.; Oh, S.T.; Foley, A.; Hughes, S.B.; Mauro, M.J.; Abdel-Wahab, O.; Press, R.D.; Viner, R.; Thompson, S.L.; Chen, Q.; et al. A Novel Germline Variant in CSF3R Reduces N-Glycosylation and Exerts Potent Oncogenic Effects in Leukemia. Cancer Res. 2018, 78, 6762-6770. [CrossRef]

9. Druhan, L.J.; McMahon, D.P.; Steuerwald, N.; Price, A.E.; Lance, A.; Gerber, J.M.; Avalos, B.R. Chronic neutrophilic leukemia in a child with a CSF3R T618I germ line mutation. Blood 2016, 128, 2097-2099. [CrossRef]

10. Maxson, J.E.; Tyner, J.W. Genomics of chronic neutrophilic leukemia. Blood 2017, 129, 715-722. [CrossRef]

11. Maxson, J.E.; Luty, S.B.; MacManiman, J.D.; Abel, M.L.; Druker, B.; Tyner, J.W. Ligand Independence of the T618I Mutation in the Colony-stimulating Factor 3 Receptor (CSF3R) Protein Results from Loss of O-Linked Glycosylation and Increased Receptor Dimerization. J. Biol. Chem. 2014, 289, 5820-5827. [CrossRef] [PubMed]

12. Elliott, M.A.; Pardanani, A.; Hanson, C.A.; Lasho, T.L.; Finke, C.M.; Belachew, A.A.; Tefferi, A. ASXL1mutations are frequent and prognostically detrimental inCSF3R-mutated chronic neutrophilic leukemia. Am. J. Hematol. 2015, 90, 653-656. [CrossRef] [PubMed]

13. Zhang, H.; Wilmot, B.; Bottomly, D.; Dao, K.-H.T.; Stevens, S.S.; Eide, C.A.; Khanna, V.; Rofelty, A.; Savage, S.; Schultz, A.R.; et al. Genomic landscape of neutrophilic leukemias of ambiguous diagnosis. Blood 2019, 134, 867-879. [CrossRef] [PubMed]

14. Dale, D.C.; Link, D.C. The Many Causes of Severe Congenital Neutropenia. N. Engl. J. Med. 2009, 360, 3-5. [CrossRef]

15. Skokowa, J.; Dale, D.C.; Touw, I.P.; Zeidler, C.; Welte, K. Severe congenital neutropenias. Nat. Rev. Dis. Prim. 2017, 3, 17032. [CrossRef]

16. Dong, F.; Brynes, R.K.; Tidow, N.; Welte, K.; Löwenberg, B.; Touw, I. Mutations in the Gene for the Granulocyte ColonyStimulating-Factor Receptor in Patients with Acute Myeloid Leukemia Preceded by Severe Congenital Neutropenia. N. Engl. J. Med. 1995, 333, 487-493. [CrossRef]

17. Skokowa, J.; Steinemann, D.; Katsman-Kuipers, J.E.; Zeidler, C.; Klimenkova, O.; Klimiankou, M.; Ünalan, M.; Kandabarau, S.; Makaryan, V.; Beekman, R.; et al. Cooperativity of RUNX1 and CSF3R mutations in severe congenital neutropenia: A unique pathway in myeloid leukemogenesis. Blood 2014, 123, 2229-2237. [CrossRef]

18. Touw, I.P.; Beekman, R. Severe congenital neutropenia and chronic neutrophilic leukemia: An intriguing molecular connection unveiled by oncogenic mutations in CSF3R. Haematologica 2013, 98, 1490-1492. [CrossRef]

19. Mitsui, T.; Watanabe, S.; Taniguchi, Y.; Hanada, S.; Ebihara, Y.; Sato, T.; Heike, T.; Mitsuyama, M.; Nakahata, T.; Tsuji, K. Impaired neutrophil maturation in truncated murine G-CSF receptor-transgenic mice. Blood 2003, 101, 2990-2995. [CrossRef]

20. Elliott, M.A.; Hanson, C.A.; Dewald, G.W.; Smoley, S.A.; Lasho, T.L.; Tefferi, A. WHO-defined chronic neutrophilic leukemia: A long-term analysis of 12 cases and a critical review of the literature. Leukemia 2004, 19, 313-317. [CrossRef]

21. Ruan, G.J.; Smith, C.J.; Day, C.; Harmsen, W.S.; Zblewski, D.L.; Alkhateeb, H.; Begna, K.; Al-Kali, A.; Litzow, M.R.; Hogan, W.; et al. A population-based study of chronic neutrophilic leukemia in the United States. Blood Cancer J. 2020, 10, 68. [CrossRef] [PubMed] 
22. Boddy, C.S.; Tan, B.T.; Aoki, J. B-lymphoblastic leukemia arising in a patient with chronic neutrophilic leukemia. Blood Adv. 2020 4, 5389-5392. [CrossRef] [PubMed]

23. Zhang, H.; Schultz, A.R.; Luty, S.; Rofelty, A.; Su, Y.; Means, S.; Bottomly, D.; Wilmot, B.; McWeeney, S.K.; Tyner, J.W. Characterization of the leukemogenic potential of distal cytoplasmic CSF3R truncation and missense mutations. Leukmia 2017, 31, $2752-2760$. [CrossRef] [PubMed]

24. Jaiswal, S.; Fontanillas, P.; Flannick, J.; Manning, A.; Grauman, P.V.; Mar, B.G.; Lindsley, R.C.; Mermel, C.H.; Burtt, N.; Chavez, A.; et al. Age-Related Clonal Hematopoiesis Associated with Adverse Outcomes. N. Engl. J. Med. 2014, 371, 2488-2498. [CrossRef] [PubMed]

25. Stoner, R.C.; Press, R.D.; Maxson, J.E.; Tyner, J.W.; Dao, K.-H.T. Insights on mechanisms of clonal evolution in chronic neutrophilic leukemia on ruxolitinib therapy. Leukemia 2019, 34, 1684-1688. [CrossRef] [PubMed]

26. Newberry, K.J.; Patel, K.; Masarova, L.; Luthra, R.; Manshouri, T.; Jabbour, E.; Bose, P.; Daver, N.; Cortes, J.; Kantarjian, H.; et al. Clonal evolution and outcomes in myelofibrosis after ruxolitinib discontinuation. Blood 2017, 130, 1125-1131. [CrossRef] [PubMed]

27. Schnittger, S.; Dicker, F.; Kern, W.; Wendland, N.; Sundermann, J.; Alpermann, T.; Haferlach, C.; Haferlach, T. RUNX1 mutations are frequent in de novo AML with noncomplex karyotype and confer an unfavorable prognosis. Blood 2011, 117, $2348-2357$. [CrossRef] [PubMed]

28. Harada, H.; Harada, Y.; Niimi, H.; Kyo, T.; Kimura, A.; Inaba, T. High incidence of somatic mutations in the AML1/RUNX1 gene in myelodysplastic syndrome and low blast percentage myeloid leukemia with myelodysplasia. Blood 2004, 103, 2316-2324. [CrossRef]

29. Takahashi, K.; Wang, F.; Morita, K.; Yan, Y.; Hu, P.; Zhao, P.; Zhar, A.A.; Wu, C.J.; Gumbs, C.; Little, L.; et al. Integrative genomic analysis of adult mixed phenotype acute leukemia delineates lineage associated molecular subtypes. Nat. Commun. 2018, 9, 2670. [CrossRef]

30. Andrews, C.; Tierens, A.; Minden, M. The genomic and biological complexity of mixed phenotype acute leukemia. Crit. Rev. Clin. Lab. Sci. 2021, 58, 153-166. [CrossRef]

31. Alexander, T.B.; Gu, Z.; Iacobucci, I.; Dickerson, K.; Choi, J.K.; Xu, B.; Payne-Turner, D.; Yoshihara, H.; Loh, M.L.; Horan, J.; et al. The genetic basis and cell of origin of mixed phenotype acute leukaemia. Nature 2018, 562, 373-379. [CrossRef] [PubMed]

32. Knoechel, B.; Bhatt, A.; Pan, L.; Pedamallu, C.S.; Severson, E.; Gutierrez, A.; Dorfman, D.M.; Kuo, F.C.; Kluk, M.; Kung, A.L.; et al. Complete hematologic response of early T-cell progenitor acute lymphoblastic leukemia to the $\gamma$-secretase inhibitor BMS-906024: Genetic and epigenetic findings in an outlier case. Mol. Case Stud. 2015, 1, a000539. [CrossRef] [PubMed] 